\title{
Una Nueva Alternativa para la Creación de Papilas en Implantes de Carga inmediata: Reporte de Caso
}

\author{
A New Alternative for the Creation of Papillae \\ in Immediate Loading Implants: Case Report
}

\author{
Britto Ebert Falcón-Guerrero
}

FALCÓN-GUERRERO, B. E. Una nueva alternativa para la creación de papilas en implantes de carga inmediata: Reporte de caso. Int. J. Odontostomat., 13(2):203-206, 2019.

RESUMEN: El objetivo de este artículo es evaluar el éxito clínico de una nueva alternativa para crear papilas en implantes de carga inmediata, que se realizó en una paciente de sexo femenino de 26 años de edad. Se inició el tratamiento con la confección de una guía quirúrgica, previo a la manipulación de los tejidos para crear las papilas peri-implantarias. En la cirugía se realizan 2 incisiones semilunares, unidas por una incisión perpendicular, las que al suturarlas sobre ellas mismas permitió crear las papilas; se instala un implante con buena estabilidad primaria y se toma la impresión para la rehabilitación. A las 24 horas se instala una corona atornillada, mostrando la presencia de las papilas de forma inmediata. Se realiza controles hasta los 20 meses de función, evidenciando buena estabilidad de las papilas mesial y distal sin presentar ningún tipo de complicación.

PALABRAS CLAVE: carga inmediata del implante dental, Implantes dentales, papila dental.

\section{INTRODUCCIÓN}

Los implantes de carga inmediata acortan el tiempo de tratamiento mediante la restauración inmediata-funcional y tienen una alta tasa de supervivencia, mientras se realice una buena selección y planificación del caso (Suarez et al., 2013; Glibert et al., 2016). Estos se instalan con una cirugía mínimamente invasiva libre de colgajo, cuyos resultados se ha optimizado con el uso de guías tomográficas (Baruffaldi et al., 2016; Cassetta \& Bellardini, 2017). Sin embargo, el resultado estético no siempre es el esperado debido a que no siempre se pueden formar las papilas, ocasionando espacios oscuros (Guarnieri et al., 2015).

El factor estético de las restauraciones implanto soportadas, debería ser idéntica a la de un diente natural; por lo que, el manejo de los tejidos blandos se ha convertido en un punto importante en la implantología y la estética gingival, siendo un factor crítico para el éxito general (Nemcovsky et al., 2000). La falta de papilas puede conducir a la impactación de alimentos, deformidades estéticas y dificultad fonética. Surgiendo un dilema clínico, cuando la papila peri implantaría tiene una altura deficiente en comparación con el diente natural, causando un espacio anti estético conocido como enfermedad del triángulo negro (Grossberg, 2001; Pradeep \& Karthikeyan, 2006).

En la actualidad, es muy importante el resultado estético gingival; por lo que, lograr crear papilas estables y predecibles es siempre un reto muy complejo, que pueden ser determinantes en el éxito de la carga inmediata (Falcón, 2016).

El objetivo de este artículo es presentar y comprobar la eficacia, mediante un reporte de caso, de una nueva alternativa para crear papilas en implantes de carga inmediata de forma sencilla, predecible y efectiva en la zona estética; siendo un caso único debido a que no existen procedimientos similares en carga inmediata, aportando una alternativa para la literatura científica. 


\section{REPORTE DEL CASO}

Paciente de 26 años, de sexo femenino, con aparente buen estado de salud general. Es evaluada en marzo del año 2017, teniendo como motivo de consulta el instalarse un implante en el margen edéntulo del diente 14.

Al examen clínico estomatológico presenta buen estado de salud periodontal y la ausencia del diente 14; al examen radiográfico se observa un margen edéntulo adecuado, sin lesiones aparentes. Después de evaluar las condiciones de la zona edéntula mediante volumetría, se decide realizar la instalación de un implante de carga inmediata previa creación de las papilas usando una nueva alternativa quirúrgica (Fig. 1).

Luego de explicarle el procedimiento y que firme un consentimiento informado; se prepara y desfocaliza a la paciente, realizando la cirugía bajo anestesia local infiltrativa y profilaxis antibiótica con $2 \mathrm{~g}$ de amoxicilna una hora previa a la cirugía. Con una hoja de bisturí \# $15-C$, se realiza dos incisiones semilunares en vestibular y palatino del área edéntula, llegando hasta unos $2 \mathrm{~mm}$ aproximadamente de los dientes naturales; luego se realiza una incisión media y perpendicular a las dos incisiones semilunares. Se eleva con cuidado dos colgajos pequeños, mesial y distal, cada uno sobre su misma base y se sutura con ácido poliglicólico 5-0. Este procedimiento, permite preservar los tejidos para crear las papilas y deja expuesta la zona de inserción del implante. Posteriormente, se prepara el lecho implantológico, insertando un implante cónico de hexágono interno de $3,3 \times 11,5 \mathrm{~mm}$, con un torque de $40 \mathrm{Nw} / \mathrm{cm}$, logrando una buena estabilidad primaria (Fig. 2).

De inmediato se toma una impresión a cubeta cerrada para elaborar la restauración definitiva y se deja con un tornillo de cicatrización. Se le indica a la paciente, ibuprofeno de $400 \mathrm{mg}$, cada $8 \mathrm{~h}$, durante 3 días y enjuagues con gluconato de clorhexidina al 0,12 $\% 2$ veces al día, por dos semanas.

A las $24 \mathrm{~h}$ posquirúrgica, se retira el tornillo de cicatrización y se instala una corona definitiva de metal-ceramica atornillada, viendo que las papilas se forman de manera inmediata, lográndose un buen resultado estético y funcional de la rehabilitación. sin que la paciente presente ningún tipo de problema o molestia, logrando satisfacer los requerimientos estéticos y funcionales de la paciente (Fig. 3).

Posteriormente, se le evalúa al mes, a los 6 meses y a los 20 meses, evidenciando una buena estabilidad de las papilas y del perfil de emergencia (Fig. 4).

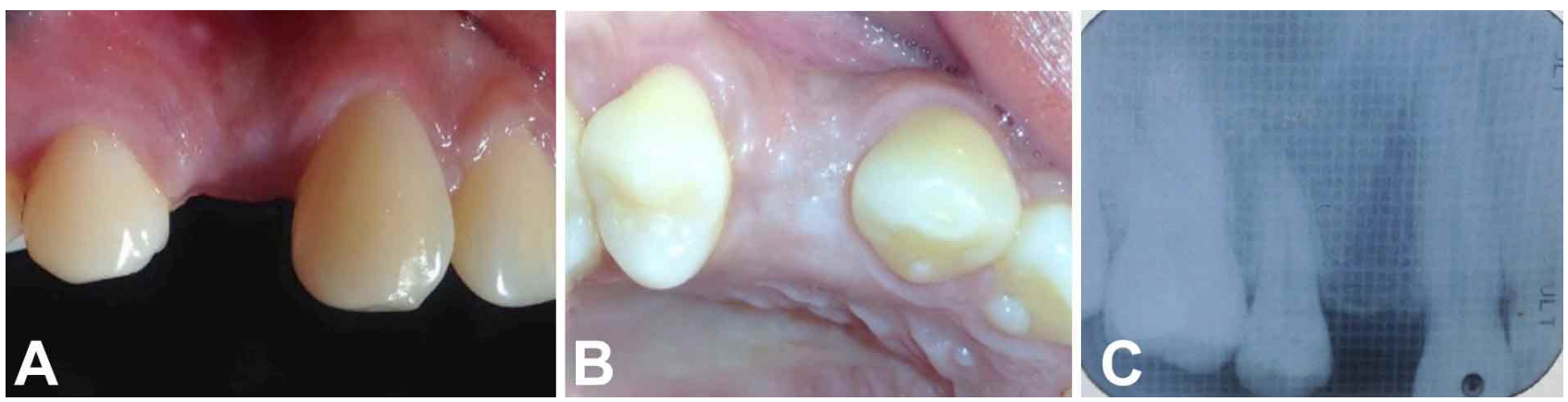

Fig. 1. Vistas preoperatorias. A: imagen vestibular. B: Imagen oclusal. C: Radiografía periapical milimetrada.
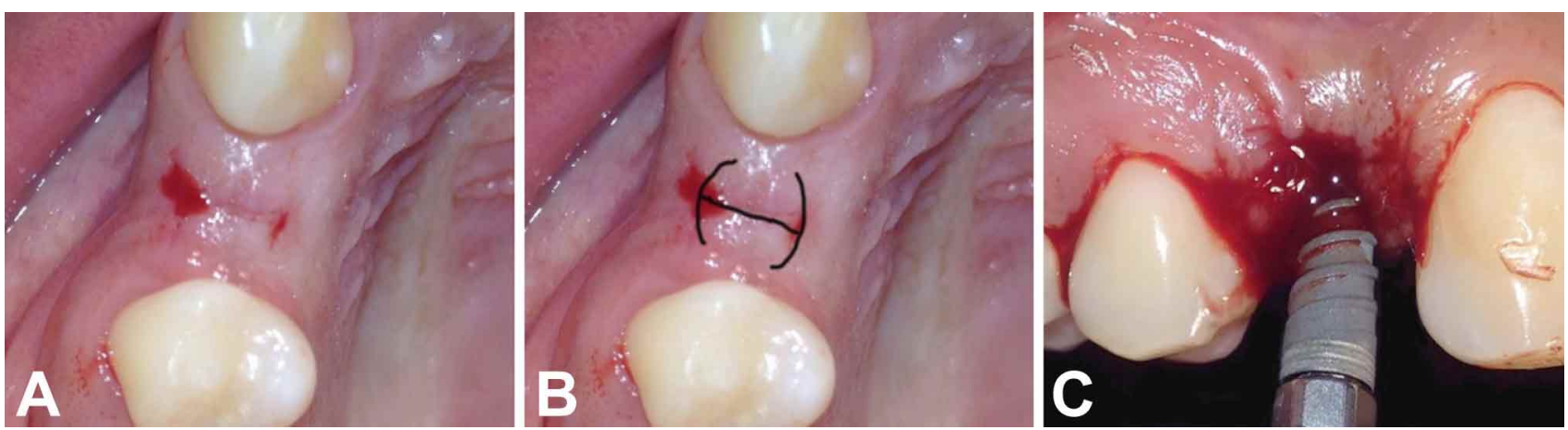

Fig. 2. A: incisión semilunar y perpendicular. B: vista grafica del diseño de la incisión. C: sutura de los mini colgajos mesial y distal e instalación inmediata del implante. 

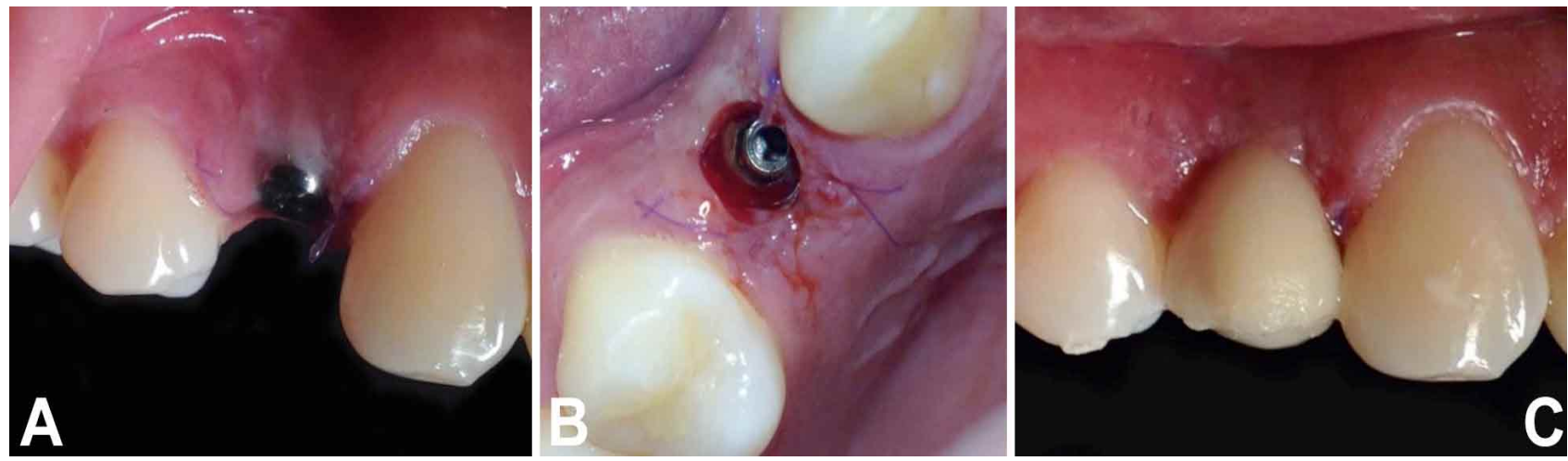

Fig. 3. Control a las 24 horas. A: imagen del tornillo de cicatrización con presencia de las nuevas papilas. B: vista oclusal donde se observa la sutura de las papilas. C: instalación de corona atornillada de carga inmediata funcional, evidenciando la creación de las nuevas papilas.
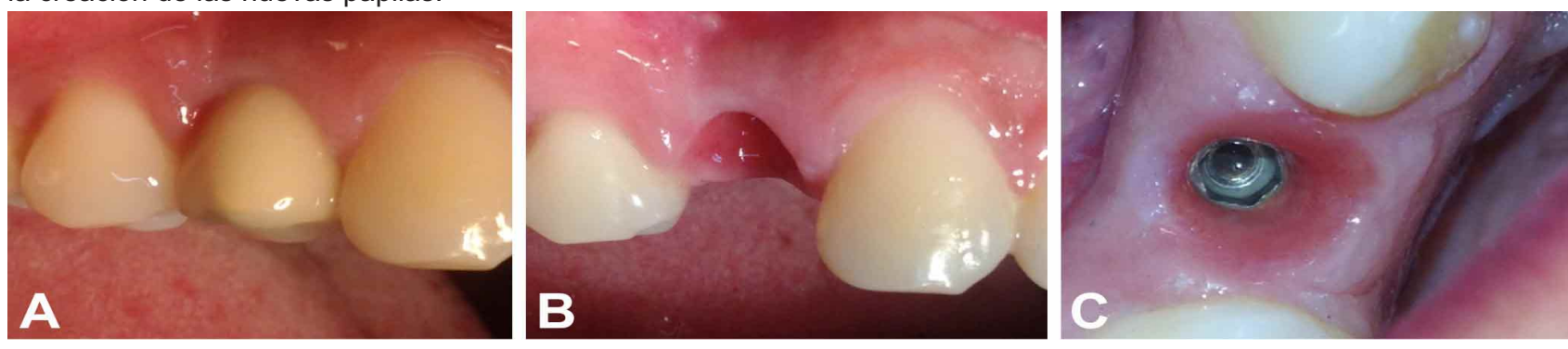

Fig. 4. Control a los 20 meses. A: imagen de la corona con papilas estables. B: vista del perfil de emergencia y papilas mesial y distal. C: imagen oclusal donde se observa una buena arquitectura gingival del perfil de emergencia.

\section{DISCUSIÓN}

El resultado estético, es un factor importante para considerar el éxito implantológico; por lo que lograrlo, siempre será un complejo reto, y así evitar que se formen triángulos oscuros que pueden ser determinantes en el resultado del tratamiento; más aún, en la zona estética de los maxilares (Guarnieri et al.; Falcón).

Los implantes de carga inmediata son tan predecibles como los de carga convencional, reportándose tasas de supervivencia del 99,1\% (Glibert et al.) y de un $96,1 \%$ a los 3 años de seguimiento (Guarnieri et al.). Estos resultados se reflejan en nuestro caso, donde el implante se mantiene estable a los 20 meses de seguimiento.

Generalmente, el procedimiento de la carga inmediata siempre se inicia con un bisturí circular o un punch sacabocado, que elimina una porción de la mucosa para así iniciar la osteotomía (Marcelis et al., s2012). Sin embargo, no siempre se logra reestablecer las papilas alrededor de los implantes, creando un problema estético (De Vico et al., 2016; Cristache \& Gurbanescu., 2017). Gjelvold et al. (2017) mencionan que el procedimiento de carga inmediata produce menos alteración de los tejidos blandos periimplantarios; pero encontraron que el índice papilar de la muestra fue estadísticamente menor en el grupo de carga inmediata. Concluyendo que tanto la carga inmediata como la tardía pueden presentar resultados satisfactorios, recién después de 12 meses de acondicionamiento.

Sin embargo, este procedimiento de acondicionamiento alarga el tiempo para llegar al resultado final y llegar a la corona definitiva; por ende, aumenta el costo y revisiones de los pacientes; lo que no sucede con esta nueva propuesta.

Jemt (1997) describe un índice para evaluar el tamaño de las papilas adyacentes a restauraciones de implantes, señalando que el índice 3 , es cuando se logra reproducir una papila que se ve armónica con los dientes naturales. Así mismo, Guarnieri et al., en un estudio para mejorar la respuesta de los tejidos bandos en la zona estética con implantes de superficie microtexturada, encontraron que solo un $52 \%$ logra un índice papilar de 3 , en la carga inmediata. 
En este reporte, aplicando esta nueva alternativa antes de instalar el implante, se logra crear las papilas mesial y distal con un índice papilar de 3 de forma inmediata, estableciendo un perfil de emergencia ideal, que guarda relación con los dientes aledaños, el cual se mantiene estable a lo largo de los 20 meses de seguimiento.

Del mismo modo la paciente no reporto molestias, dolor, inflamación; sintiéndose cómoda desde el posoperatorio inmediato, manifestando incluso que sí se volvería a hacer este tipo de cirugía, de darse el caso, por el resultado estético y funcional inmediato.

En este sentido, se ha informado que la satisfacción del paciente después de la carga inmediata y carga convencional, es alta y no se observan diferencias entre los grupos, aunque se menciona que, en la carga convencional, el tratamiento es muy largo (den Hartog et al., 2011).

\section{CONCLUSIÓN}

La técnica descrita es una buena alternativa clínica para lograr la creación de las papilas en implantes de carga inmediata, es simple y acorta el tiempo de tratamiento, reduce los costos, brinda mayor confort y el resultado estético es efectivo inmediatamente, optimizando el resultado en el sector estético de los pacientes.

FALCÓN-GUERRERO, B. E. A new alternative for the creation of papillae in immediate loading implants: case report. Int. J. Odontostomat., 13(2):203-206, 2019.

ABSTRACT: The aim of this paper is to evaluate the clinical success of a new alternative to create papillae in immediate load implants, which was performed in a 26-yearold female patient. The treatment was started with the preparation of a surgical guide, prior to the manipulation of tissues to create the peri-implant papillae. In the surgery, two semilunar incisions are made, joined by a perpendicular incision, which when sutured on them allowed to create the papillae. An implant with good primary stability is installed and the impression is taken for rehabilitation. At $24 \mathrm{~h}$ a screwed crown is installed, showing the presence of the papillae immediately. Controls are performed up to 20 months of function, demonstrating good stability of the mesial and distal papillae without presenting any type of complication.

KEY WORDS: immediate dental implant loading, dental implants, dental papilla.

\section{REFERENCIAS BIBLIOGRÁFICAS}

Baruffaldi, A.; Poli, P. P.; Baruffaldi, A.; Giberti, L.; Pigozzo, M. \& Maiorana, C. Computer-aided flapless implant surgery and immediate loading. A technical note. Oral Maxillofac. Surg., 20(3):313-9, 2016.

Cassetta, M. \& Bellardini, M. How much does experience in guided implant surgery play a role in accuracy? A randomized controlled pilot study. Int. J. Oral Maxillofac. Surg., 46(7):922-30, 2017.

Cristache, C. M. \& Gurbanescu, S. Accuracy evaluation of a stereolithographic surgical template for dental implant insertion using 3D superimposition protocol. Int. J. Dent., 2017:4292081, 2017.

De Vico, G.; Ferraris, F.; Arcuri, L.; Guzzo, F. \& Spinelli, D. A novel workflow for computer guided implant surgery matching digital dental casts and CBCT scan. Oral Implantol. (Rome), 9(1):33-48, 2016.

den Hartog, L.; Raghoebar, G. M.; Stellingsma, K.; Vissink, A. \& Meijer, H. J. Immediate non-occlusal loading of single implants in the aesthetic zone: a randomized clinical trial. J. Clin. Periodontol., 38(2):186-94, 2011.

Falcón, B. Effectiveness of a new surgical approach: "Tinkiy," for reconstruction of peri implant papilla and the treatment of gingival recession. J. Implant Adv. Clin. Dent., 8(1):45-53, 2016.

Gjelvold, B.; Kisch, J.; Chrcanovic, B. R.; Albrektsson, T. \& Wennerberg, A. Clinical and radiographic outcome following immediate loading and delayed loading of single-tooth implants: Randomized clinical trial. Clin. Implant Dent. Relat. Res., 19(3):549-58, 2017.

Glibert, M.; De Bruyn, H. \& Östman P. O. Six-year radiographic, clinical, and soft tissue outcomes of immediately loaded, straight-walled, platform-switched, titanium-alloy implants with nanosurface topography. Int. J. Oral Maxillofac. Implants, 31(1):167-71, 2016.

Grossberg, D. E. Interimplant papilla reconstruction: assessment of soft tissue changes and results of 12 consecutive cases. J. Periodontol., 72(7):958-62, 2001.

Guarnieri, R.; Grande, M.; Ippoliti, S.; Iorio-Siciliano, V.; Riccitiello, F. \& Farronato, D. Influence of a laser-lok surface on immediate functional loading of implants in single-tooth replacement: threeyear results of a prospective randomized clinical study on soft tissue response and esthetics. Int. J. Periodontics Restorative Dent., 35(6):865-75, 2015.

Jemt, T. Regeneration of gingival papillae after single-implant treatment. Int. J. Periodontics Restorative Dent., 17(4):326-33, 1997.

Marcelis, K.; Vercruyssen, M.; Naert, I.; Teughels, W. \& Quirynen, M. Model-based guided implant insertion for solitary tooth replacement: a pilot study. Clin. Oral Implants Res., 23(8):999-1003, 2012.

Nemcovsky, C. E.; Moses, O. \& Artzi, Z. Interproximal papillae reconstruction in maxillary implants. J. Periodontol., 71(2):308-14, 2000.

Pradeep, A. R. \& Karthikeyan, B. V. Peri-implant papilla reconstruction: realities and limitations. J. Periodontol., 77(3):534-44, 2006.

Suarez, F.; Chan, H. L.; Monje, A.; Galindo-Moreno, P. \& Wang, H. L. Effect of the timing of restoration on implant marginal bone loss: a systematic review. J. Periodontol., 84(2):159-69, 2013.

Dirección para correspondencia:

Britto Ebert Falcón-Guerrero

Universidad Nacional Jorge Basadre Grohmann

Dirección: Av. Tarapacá \# 350

Tacna

PERÚ

Recibido : 22-11-2018

Aceptado: 20-02-2019

Email: artdent2000@hotmail.com 